\title{
Buckling optimization of composite laminates using a hybrid algorithm under Puck failure criterion constraint
}

\author{
H Arda Deveci ${ }^{1,2}$, Levent Aydin ${ }^{3}$ and H Seçil Artem'
}

\begin{abstract}
In this study, an optimization procedure is proposed to find the optimum stacking sequence designs of laminated composite plates in different fiber angle domains for maximum buckling resistance. A hybrid algorithm combining genetic algorithm and trust region reflective algorithm is used in the optimization to obtain higher performance and improve the quality of solutions. As a novelty, Puck fiber and inter-fiber failure criteria are directly implemented to the optimization problems as nonlinear function constraints, which have allowed more consistent and feasible results. The performance of the hybrid algorithm is demonstrated by comparing with the individual performances of genetic and trust region reflective algorithms via test problems from the literature. Also, a study is performed to exhibit the effectiveness of the selected failure criterion as constraint among the other common criteria. The proposed procedure is used to solve many problems including various design considerations. The results indicate that reliable stacking sequence designs can be achieved in specific configurations even for the composite plates subjected to superior buckling loads when Puck physically based (3D) failure theory is considered as a first ply failure constraint in the buckling optimization.
\end{abstract}

\section{Keywords}

Buckling, optimization, hybrid algorithm, composite laminate, Puck failure criterion

\section{Introduction}

Fiber-reinforced composites are commonly used in many engineering applications such as aircraft, aerospace, automotive, and marine due to their high specific strength, stiffness, lightweight, corrosion resistance and high durability as compared to other traditional materials. Besides the inherent advantages of composites, the nature of fiber-reinforced composites enables the unique opportunity of tailoring their properties according to design requirements for a given application. The full potential of laminated composite plates can be achieved by searching the optimum stacking sequence designs. ${ }^{1,2}$

Design of composite structures is treated as a global optimization problem including multiple local optima and complex design space. Gradient-based optimization methods are not appropriate for discrete stacking sequence optimization problems of laminated composite plates. In gradient methods, the solution's success highly depends on choosing a starting point which is sufficiently close to the global optimum; otherwise, it may easily get stuck into any local optimum. These methods do not find large application area to composite lay-up design, since gradient information is often either unavailable or computationally expensive to determine. Hence, stochastic optimization methods are more suitable for composite lay-up design due to their capabilities of handling a mixture of continuous and discrete variables, and finding the global optimum

\footnotetext{
'Department of Mechanical Engineering, Izmir Institute of Technology, Izmir, Turkey

${ }^{2}$ Department of Mechanical Engineering, Erzincan University, Erzincan, Turkey

${ }^{3}$ Department of Mechanical Engineering, Izmir Katip Çelebi University, Izmir, Turkey
}

\section{Corresponding author:}

H Seçil Artem, Department of Mechanical Engineering, Izmir Institute of Technology, Gulbahce Kampusu, 35430-Urla, Izmir, Turkey.

Email: secilartem@iyte.edu.tr 
of a multi-modal objective function. ${ }^{3,4}$ Genetic Algorithm (GA) is the most commonly used stochastic optimization method in design and optimization of composite laminates. ${ }^{5-13}$ GAs are also well suited for stacking sequence optimization in which they can produce alternative optima in repeated runs due to their random nature. ${ }^{14}$

Buckling should be considered as a critical failure mode for thin and large composite plates subjected to compressive in-plane loads. Determination of buckling load capacity of such composite plates is crucial for design of composite structures. ${ }^{15}$ Therefore, buckling load maximization problem of composite plates has drawn attention of researchers for years. As one of the pioneering studies in this subject, the stacking sequence design of a composite laminate for buckling load maximization using GA is treated by Le Riche and Haftka. $^{5}$ Furthermore, the same researchers investigated the minimum thickness design of composite laminated plates with an improved GA. ${ }^{6}$ Kim and Lee ${ }^{10}$ studied the GA optimization in order to obtain the worst and best designs of composite laminates with continuous fiber orientations for the maximum buckling load. Karakaya and Soykasap ${ }^{13}$ maximized the critical buckling load factor for different design cases in order to design the optimum composite plates with conventional fiber angles. Stacking sequence optimization of laminated composites for maximization of buckling load factor has also been considered with other stochastic optimization methods in the literature such as generalized pattern search algorithm, ${ }^{13}$ simulated annealing (SA), ${ }^{14}$ Tabu search, ${ }^{16}$ scatter search, ${ }^{17}$ ant colony optimization (ACO) $)^{18,19}$ algorithms.

In addition to standard meta-heuristic algorithms, in the literature, buckling load factor maximization problems are also considered with various hybrid algorithms (HAs) to find the optimum stacking sequence designs. HAs take advantage of the best features of different algorithms by combining them in order to derive a relatively superior algorithm. Examples of these HAs include Tabu search embedded SA algorithm, ${ }^{20-22}$ particle swarm optimization algorithm with discrete-variable-depth neighborhood search algorithm, ${ }^{23}$ shuffled frog-leaping algorithm hybridized with a customized neighborhood search algorithm, ${ }^{24}$ and Tabu embedded ACO. $^{25}$ It is reported that these HAs typically exhibit better or at least comparable performance on finding the optimum results in comparison to standard metaheuristic algorithms in the literature.

In addition to that buckling is a critical failure mode in composite plates subjected to high in-plane loads, the first ply failure is also a significant factor to be considered in design of laminated composite plates. In many applications, composite structures are considered to be failed under first ply failure. On the other hand, progressive failure is mainly important for postbuckling analysis of composite plates. Only initial failure behavior is considered in this study. Numerous failure theories have been proposed with the aim of explaining the actual failure behavior of composite laminates. It is possible to categorize these failure criteria of composite plates into three groups: limit or non-interactive theories such as Maximum stress or maximum strain, interactive theories such as TsaiHill, Tsai-Wu (TW) or Hoffman, and partially interactive or failure mode-based (physically based) theories such as Puck. ${ }^{12}$ Physically based failure criteria provide realistic information related to crack initiation, propagation, and the risk of ultimate failure of laminated composite structures. ${ }^{26}$ It is also a known fact that using multiple failure criteria simultaneously for optimization problems involving composites can be beneficial. ${ }^{27}$ However, considering the literature, the number of optimization studies in which physically based failure criteria are used as strength constraint is considerably few. This critical fact has begun to be taken into account by some researchers. For example, Lopez et al. $^{12}$ proposed a stacking sequence optimization problem so as to minimize the weight and cost parameters under the first ply failure constraint using Puck failure criteria (PFC). Sebaey et al. ${ }^{19}$ maximized the critical buckling load for both biaxial compression and tension cases considering the first ply failure as strength constraint. On the other hand, it is difficult to select the appropriate failure theory for use in a given design situation as the validity and applicability of a failure criterion are directly based on the convenience of application and agreement with reliable experimental data. ${ }^{12}$ In this respect, a "Worldwide Failure Exercise" is organized and led by Soden et al. ${ }^{28}$ with the aim of evaluating the predictive capabilities of generally acceptable failure theories in the research community. The participants are requested to conduct many tests in various compressive and/or tensile, uniaxial and/or biaxial loading cases. The results of the exercise show that the Puck failure theory exhibits better robustness and accuracy performances considering the experimental results in the overall ranking as compared to many others. ${ }^{29}$ Mainly, it is important for the present study that Puck failure theory has been validated for tests with biaxial compressive loading.

The composite plates subjected to high compressive loads may fail due to ply fracture before buckling occurs. Therefore, strength of laminate should be considered in optimization problems where buckling is critical. In the literature, although there are many studies regarding buckling load capacity maximization of composite plates, very few researches ${ }^{12,19}$ have taken into account the strength requirements. Furthermore, direct implementation of Puck failure criterion into 
the optimization as nonlinear constraint has not been practiced so far. Besides, in the view of the HA studies previously mentioned, a hybrid approach to the buckling optimization of composite plates can provide improved designs. In this regard, the present study has been organized to obtain optimum integer and discrete stacking sequences of laminated composite plates by a HA for maximum buckling load capacity considering first ply failure as constraint. Classical lamination theory and linear buckling analysis are used to generate the objective function necessary for optimization. Unlike other studies in the literature, Puck fiber and inter-fiber failure (IFF) criteria are implemented to the optimization as nonlinear constraints, which have allowed more consistent and accurate results.

\section{Failure analysis}

The design of laminated composite plates, according to the demands such as buckling resistance and/or weight minimization indispensably, requires strength constraints. With this aspect, the research field of failure criteria for fiber-reinforced composites has drawn attention of many researchers over the last few decades. More than 40 failure criteria have been developed for laminated composite materials thus far. Among them, the maximum strain criterion is the most commonly used in the stacking sequence optimization studies, and the TW criterion is the most commonly used in the industry. ${ }^{19}$ The use of criteria that closely reflect the actual behavior of the laminated composites under study is critical. Many studies about optimum design (OD) of composites consider common failure criteria based on von Mises or Hill yield criteria, which are more suitable for ductile materials. Essentially, due to the fact that the failure of composite materials is similar to brittle behavior, it is more appropriate to use the failure criteria conformed to materials that show brittle fracture behavior such as Mohr's criterion. The Puck failure theory of composite plates relies on Mohr's criterion in which fracture is provoked exclusively by the stresses acting on the fracture plane. ${ }^{12}$ The detailed assessment can be found in literature. ${ }^{26,30}$ In the following subsections two main failure types of PFC fiber failure (FF) and IFF are introduced.

\section{Puck FF}

Failure analysis of laminated composite plates generally depends on the stresses in each lamina in the principal material coordinates. In the Puck FF, it is assumed that FF in a unidirectional composite under multiaxial stresses occurs at the same stress value which leads to failure of the fibers under a uniaxial stress. ${ }^{12}$ According to the theory, ${ }^{26} \mathrm{FF}$ occurs,

For tensile stresses

$$
\begin{aligned}
& \frac{1}{\varepsilon_{1 T}}\left(\varepsilon_{1}+\frac{v_{f 12}}{E_{f 1}} m_{\sigma f} \sigma_{2}\right)=1 \\
& \quad \text { valid for }\left(\varepsilon_{1}+\frac{v_{f 12}}{E_{f 1}} m_{\sigma f} \sigma_{2}\right) \geq 0
\end{aligned}
$$

For compressive stresses

$$
\begin{gathered}
\frac{1}{\varepsilon_{1 C}}\left|\left(\varepsilon_{1}+\frac{v_{f 12}}{E_{f 1}} m_{\sigma f} \sigma_{2}\right)\right|+\left(10 \gamma_{21}\right)^{2}=1 \\
\text { valid for } \left.\left(\varepsilon_{1}+\frac{v_{f 12}}{E_{f 1}} m_{\sigma f} \sigma_{2}\right)\right)<0
\end{gathered}
$$

where $\varepsilon_{1 T}$ and $\varepsilon_{1 C}$ are tensile and compressive failure strains in the fiber direction, respectively; $\varepsilon_{1}$ is the normal strain occurring in the fiber direction; $\sigma_{1}$ and $\sigma_{2}$ are normal stresses in the fiber and transverse directions, respectively; $v_{f 12}$ is the Poisson's ratio of the fibers; $m_{\sigma f}$ is for a stress magnification effect caused by the different moduli of the fibers and matrix in the $\sigma_{2}$ direction $\left(m_{\sigma f} \approx 1.3\right.$ for glass fiber, $m_{\sigma f} \approx 1.1$ for carbon fiber); $E_{f 1}$ is the Young's modulus of the fiber; $\gamma_{21}$ is the shear strain in the plane $1-2$ and $\left(10 \gamma_{21}\right)^{2}$ is an empirical shear correction.

\section{Puck IFF}

IFF is based on a physical basis which provides further information about the angle of fracture $\left(\theta_{f p}\right)$. For IFF criterion, the axes of the failure plane and the related stresses are presented in Figure 1.

IFF has three failure modes which are referred to as A, B and C. ${ }^{26}$ The fracture modes with their boundaries are shown in Figure 2.

Failure Mode A occurs when the lamina is subjected to tensile transverse stress; however, failure Mode B and Mode $\mathrm{C}$ are related to compressive transverse stress. The division of the modes is determined by the approach that a tensile stress $\sigma_{n}>0$ leads to fracture while a compressive stress impedes shear fracture. For $\sigma_{n}<0$, the shear stresses $\tau_{n t}$ and/or $\tau_{n 1}$ have to cause fracture against an additional fracture resistance increases with increasing $\left|\sigma_{n}\right|$. The difference between Mode $\mathrm{B}$ and $\mathrm{C}$ is related to their fracture angles. In Mode B, the fracture angle of the lamina is assumed as $0^{\circ}$; however, it corresponds to a different value for Mode C. Essentially, Mode C is more critical in comparison with modes $\mathrm{A}$ and $\mathrm{B}$ because it produces inclined cracks which may lead to delamination of layers. As well, an inclined wedge-shaped inter-fiber 


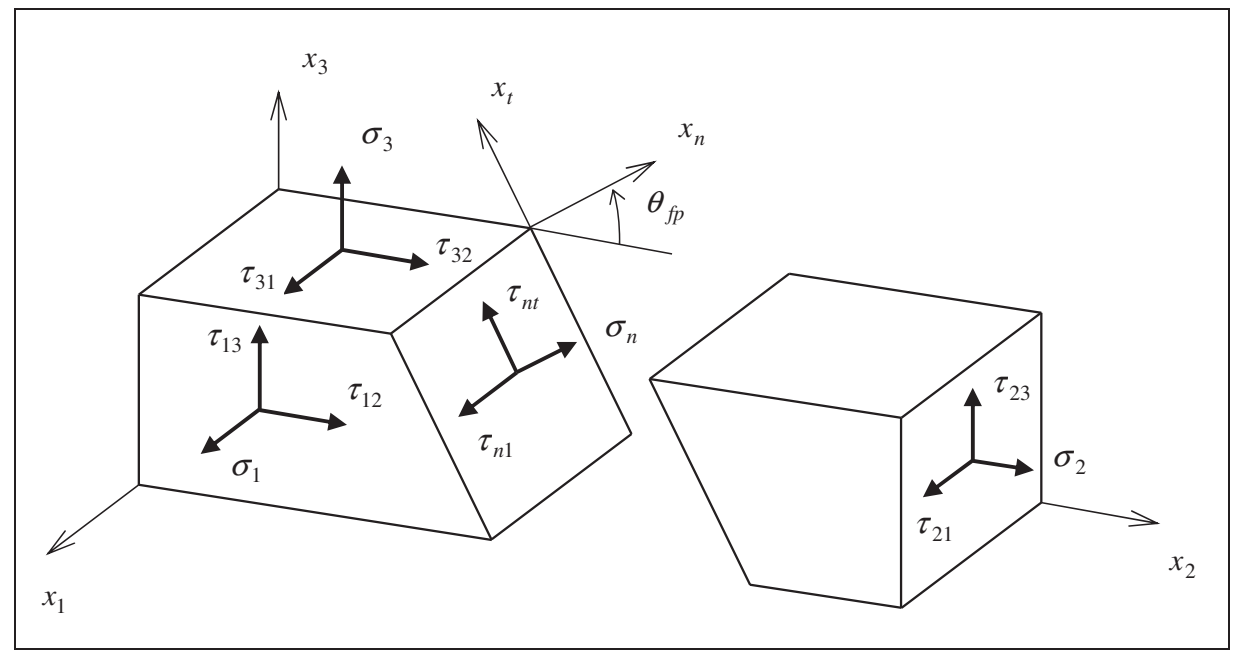

Figure I. Three-dimensional stresses on a unidirectional composite element.

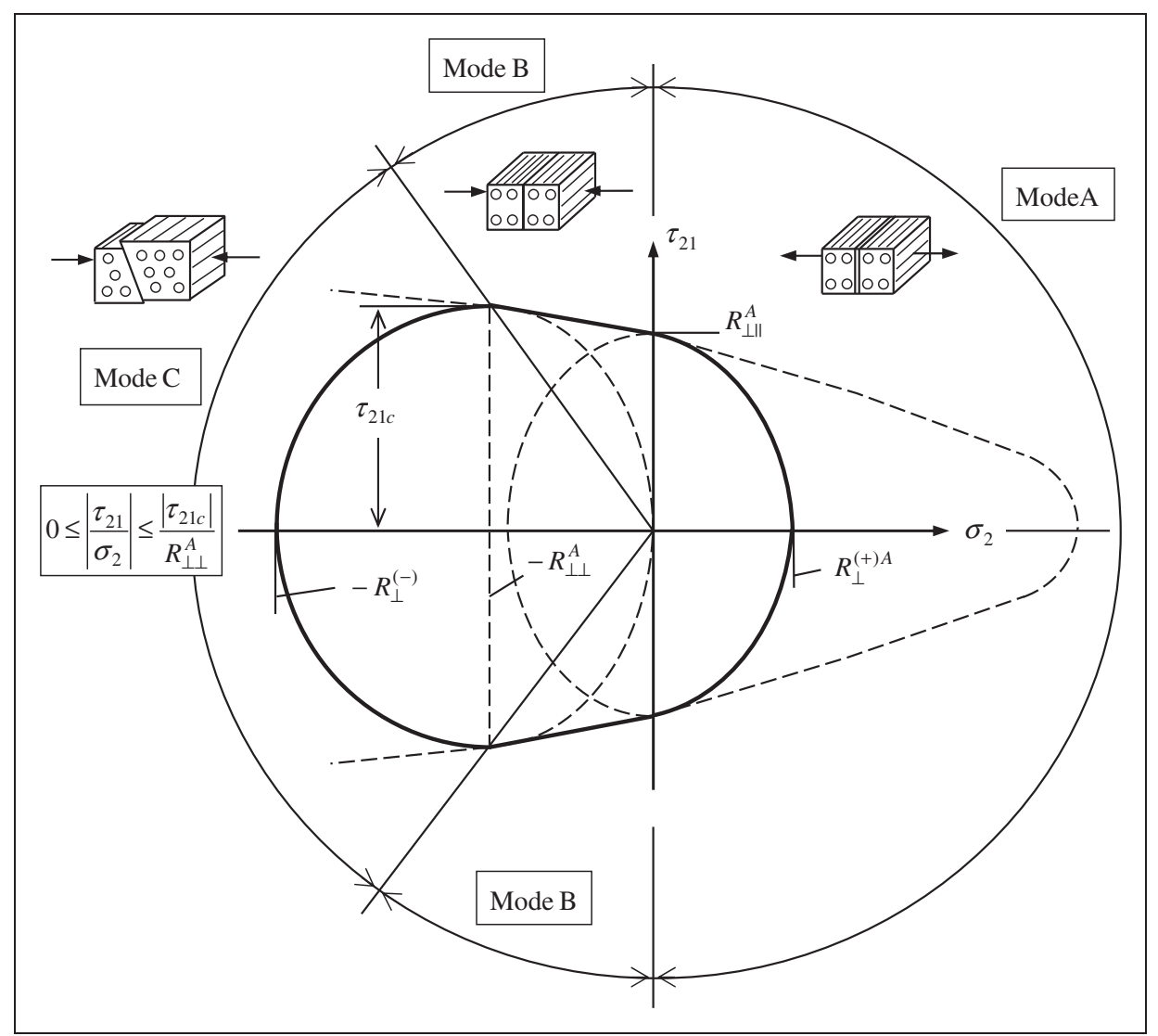

Figure 2. The three different fracture modes (A, B and C) for the PFC. ${ }^{26}$ 
crack can provoke ultimate failure of the laminate. Since the laminated plates considered in our study are subjected to in-plane compressive loads, only Modes B and $\mathrm{C}$ are taken into account.

The expressions for the related modes are as follows

$$
\begin{aligned}
& \text { Mode B }\left(\theta_{f p}=0^{\circ}\right) \\
& \frac{1}{S_{21}}\left(\sqrt{\tau_{21}^{2}+\left(p_{\perp \|}^{(-)} \sigma_{2}\right)^{2}}+p_{\perp \|}^{(-)} \sigma_{2}\right)=1-\left|\frac{\sigma_{1}}{\sigma_{1 D}}\right| \text { if } \\
& \sigma_{2}<0 \text { and } 0 \leq\left|\frac{\sigma_{2}}{\tau_{21}}\right| \leq \frac{R_{\perp \perp}^{A}}{\left|\tau_{21 c}\right|}
\end{aligned}
$$

Mode C $\left(\theta_{f p}=\arccos \sqrt{\frac{f_{w} R_{\perp \perp}^{A}}{\left(-\sigma_{2}\right)}}\right)$

$$
\begin{aligned}
& {\left[\left(\frac{\tau_{21}}{2\left(1+p_{\perp \perp}^{(-)}\right) S_{21}}\right)^{2}+\left(\frac{\sigma_{2}}{Y_{C}}\right)^{2}\right] \frac{Y_{C}}{\left(-\sigma_{2}\right)}=1-\left|\frac{\sigma_{1}}{\sigma_{1 D}}\right| \text { if }} \\
& \sigma_{2}<0 \text { and } 0 \leq\left|\frac{\tau_{21}}{\sigma_{2}}\right| \leq \frac{\left|\tau_{21 c}\right|}{R_{\perp \perp}^{A}}
\end{aligned}
$$

where the weakening factor $f_{w}=\left(0.9 f_{E(F F)}\right)^{n}$ is for the degradation effect of $\sigma_{1}$ and $n$ depends on the matrix of the laminate (for example, $n=6$ for epoxy). The definitions for parameters $p_{\perp \|}^{(-)}, p_{\perp \perp}^{(-)}, R_{\perp \perp}^{A}, \tau_{21 c}$ can be found in Puck and Schürmann.

\section{Optimization}

Composite laminate design problems cannot be handled with the traditional optimization techniques because their derivative calculations or approximations are almost impossible to achieve and are required great computational effort. ${ }^{31}$ As an evolutionary algorithm technique, GA provides significant advantages over traditional gradient-based optimization routines, such as robustness to problem complexity and the ability to discover easily global optimum rather than local stationary optima. ${ }^{1}$ Nevertheless, standard GAs have the disadvantages of (i) slow convergence rates when they work with complicated or time-consuming objective functions, (ii) finding local optima and (iii) the lack of cooperation between populations. However, if a gradient-based local optimum algorithm with an initial guess near the optima is used, it converges faster to a solution. In order to overcome these weak points in the heuristic (e.g. GAs) and gradient-based approaches, some hybrid methods have been preferred by the researchers ${ }^{32,33}$ and solved by taking advantages of each positive element of these approaches. For example, by combining the advantages of both methods: first the GA is applied to obtain a point close to the global minimum, and then the gradient-based method is applied to refine and improve the result obtained by the GA, global convergence can be guaranteed. In this step, selection of gradient-based local optimum algorithm is critical. In the study by Ayesta et al., ${ }^{34}$ a comparison among gradient-based methods has been presented. Then, a comparison among heuristic methods has been carried out, and finally the chosen hybrid method combining the best of each group has been compared with the results obtained using either a gradient-based method or a heuristic method. They concluded that, in order to obtain best results, a hybrid method composed by a GA to find an initial point and a trust region reflective method to refine this value has been chosen. After literature survey on the HAs, we decided to consider in this study the combination of GA and a trust region method as a HA to achieve a high accuracy and a moderate computational effort in our results. The GA is used for obtaining the initial predictions of the parameters, and then the TRRA is used to obtain the values of the parameters corresponding to the global minimum. Thus, it is not necessary that the GA values of the parameters are those corresponding to a global minimum as long as the values obtained are those in the close neighborhood of the global minimum. ${ }^{35}$

Any information on GA is not given here due to the fact that GA has been frequently used in laminate optimization problems. ${ }^{5-13}$ However, relatively new algorithm trust region reflective algorithm (TRRA) will be briefly introduced.

TRRA has been commonly used in the optimization field due to their fast convergence abilities. In general, TRRA searches the solution around a point in a twodimensional region of trust in which the optimization problem is approximated by a quadratic sub-problem, and then transformed to the original problem. This resulting sub-problem falls into the class of quadratic problems and their solutions can be found effectively by using some exact and approximate methods, which leads to the fast convergence to the global optimum. ${ }^{36}$ Step-by-step mathematical procedure will be outlined as follows: ${ }^{37,38}$

Step 1. Assume $f(x), x \in R^{n}$ is the function to be minimised with $x$ as a vector of required variables. ( $l \leq x \leq u$ where $l$ and $u$ denotes vectors of lower and upper bounds, respectively.) 


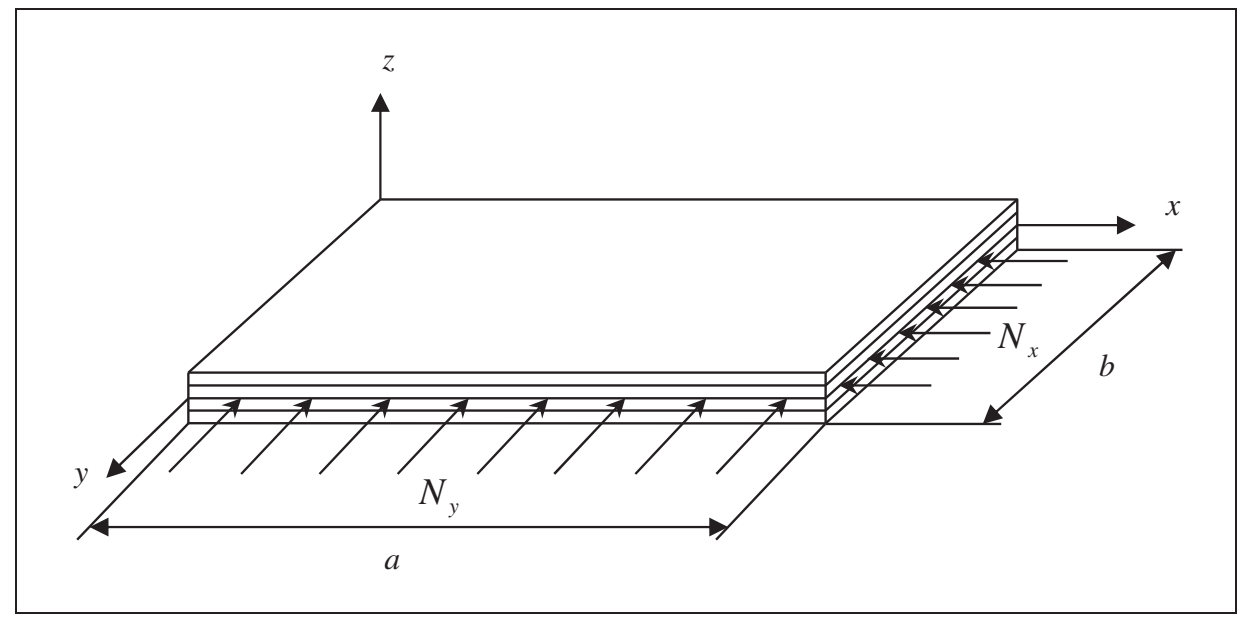

Figure 3. Laminated composite plate subjected to compressive in-plane loads.

Step 2. Choose starting vector parameters $\bar{\Delta}$, $\Delta_{0} \in(0, \bar{\Delta}), 0<\eta_{1} \leq \eta_{2}<1,0<\gamma_{1}<1<\gamma_{2}, \varepsilon>0$, and $k=0$. (where $\bar{\Delta}$ is the overall bound for the trust region radius $\Delta_{k}, \varepsilon$ is the tolerance.)

Step 3. Compute 2D sub-space $S$, in order to find the vectors $s_{1}$ and $s_{2}$.

Step 4. Approximately solve the general trust-region sub-problem to determine $s_{k}$

$$
\min _{s}\left\{q_{k}(s)=f_{k}+s^{T} g_{k}+\frac{1}{2} s^{T} H_{k} s,\left\|D_{k} s\right\| \leq \Delta_{k}\right\}
$$

where $H$ is the Hessian matrix, $g$ is the gradient of $f$ at the current point $x, D_{k}$ is a diagonal scaling matrix, $\Delta_{k}$ is the positive scalar radius of trust region, and $k$ is iteration number. The applied solution approach is to restrict the trust region sub-problem to a twodimensional subspace $S$ and the determination of the subspace. The detailed explanation on the approximate solution of the trust region sub-problems can be found in literature. ${ }^{39,40}$

Step 5. Compute $r_{k}=\frac{f\left(x_{k}\right)-f\left(x_{k}+s_{k}\right)}{q_{k}(0)-q_{k}\left(s_{k}\right)}$, Set

$$
x_{k+1}=\left\{\begin{array}{c}
x_{k}+s_{k}, \text { if } r_{k} \geq \eta_{1} \\
x_{k}, \text { otherwise }
\end{array}\right.
$$

Step 6. Adjust the trust region radius $\Delta_{k+1}$ if $r_{k}<\eta_{1}, \Delta_{k+1} \in\left(0, \gamma_{1} \Delta_{k}\right)$ (shrink trust region) if $\eta_{1} \leq r_{k}<\eta_{2}, \quad \Delta_{k+1} \in\left[\gamma_{1} \Delta_{k}, \Delta_{k}\right]$ (shrink or accept the previous trust region) if $r_{k} \geq \eta_{2}$ and $\left\|s_{k}\right\|=\Delta_{k}$, $\Delta_{k+1} \in\left[\Delta_{k}, \min \left\{\gamma_{2} \Delta_{k}, \overline{\Delta_{k}}\right\}\right]$ (Enlarge trust region).

Step 7. If $\left\|g:=\nabla f\left(x_{k}\right)\right\| \leq \varepsilon$, the solution is converge and the algorithm stops. Otherwise, set $k=k+1$ and update the quadratic model, then go to Step 3. It should be noted that the very important issue in a trust-region algorithm is how to solve the trust region sub-problem.

\section{Problem definition}

In this study, 64-layered rectangular carbon/epoxy composite plates simply supported on four sides with length of $a$ and width of $b$ are considered for optimization. The composite plates are subjected to in-plane biaxial compressive loads per unit length $N_{x}$ and $N_{y}$, as shown in Figure 3.

The laminated composite plate will buckle into $m$ and $n$ half waves in the $x$ and $y$ directions when the amplitude parameter reaches a value $\lambda_{b}$ given by

$$
\lambda_{b}=\frac{\pi^{2}\left[D_{11}\left(\frac{m}{a}\right)^{4}+2\left(D_{12}+2 D_{66}\right)\left(\frac{m}{a}\right)^{2}\left(\frac{n}{b}\right)^{2}+D_{22}\left(\frac{n}{b}\right)^{4}\right]}{N_{x}\left(\frac{m}{a}\right)^{2}+N_{y}\left(\frac{n}{b}\right)^{2}}
$$

where $D_{i j}$ are bending stiffness coefficients, and $\lambda_{b}$ is called buckling load factor. ${ }^{41,42}$

The smallest value of $\lambda_{b}$ under appropriate $m$ and $n$ values indicates the critical buckling load factor $\lambda_{c b}$, and it determines the maximum load which the laminate can withstand without buckling and varies with the plate aspect ratio, loading ratio and material. In order to avoid any immediate failure, $\lambda_{c b}$ should be greater than the value of one. It is reported that the critical values of $m$ and $n$ are small unless the plate has a very high aspect ratio or extreme ratios of $D_{i j}{ }^{43}$ Therefore, in this study, the values of $m$ and $n$ are taken to be 1 or 2 in order to result in a good estimation of buckling load capacity. Thus, the smallest of $\lambda_{b}(1,1)$, $\lambda_{b}(1,2), \lambda_{b}(2,1)$ and $\lambda_{b}(2,2)$ yields $\lambda_{c b}{ }^{44}$

For the plate in question, layer thickness is $0.25 \mathrm{~mm}$ and the length $a$ equals to $0.508 \mathrm{~m} . N_{x}$ is taken as $1000 \mathrm{~N} / \mathrm{mm}, \quad 3000 \mathrm{~N} / \mathrm{mm}, \quad 5000 \mathrm{~N} / \mathrm{mm}$ and $10,000 \mathrm{~N} /$ $\mathrm{mm}$. The plate design is studied under load ratios; $N_{x} / N_{y}=1 / 2, \quad N_{x} / N_{y}=1, \quad N_{x} / N_{y}=2, \quad N_{x} / N_{y}=4$, and aspect ratios; $a / b=1, a / b=2, a / b=4$, 
Table I. Material properties of the laminae. ${ }^{12}$

\begin{tabular}{ll}
\hline Strength properties & $X_{T}=2062 \mathrm{MPa}, X_{C}=I 70 I \mathrm{MPa}$, \\
& $Y_{T}=70 \mathrm{MPa}, Y_{C}=240 \mathrm{MPa}$, \\
& $S_{I 2}=105 \mathrm{MPa}, \varepsilon_{\mid T} 0.0175, \varepsilon_{\mid C}=0.014$ \\
Fiber properties & $E_{f \mid}=230000 \mathrm{MPa}, v_{f \mid 2}=0.23$ \\
PFC parameters & $m_{o f}=1 . I_{\perp \|}^{(-)}=0.25, p_{\perp \|}^{(+)}=0.3$ \\
\hline
\end{tabular}

$a / b=1 / 2 . N_{y}$ and $b$ are calculated from the load ratios and aspect ratios accordingly. The elastic material properties of the layers are taken as: longitudinal modulus $E_{1}=116,600 \mathrm{MPa}$, transverse modulus $E_{2}=7673 \mathrm{MPa}$, in-plane shear modulus $G_{12}=4173$ $\mathrm{MPa}$, and Poisson's ratio $v_{12}=0.27 .{ }^{12}$ Strength properties, elastic fiber properties and PFC parameters with regard to the laminae are given in Table 1.

The optimization problem can be defined as

Maximize: $\quad \lambda_{c b}\left(\theta_{k}\right), \quad \theta_{k} \in[-90,90] \vee\left\{0_{2}, \pm 45,90_{2}\right\}$, $k=1, \ldots, 64$

Constraints: First ply failure (PFC)

$\left\{f_{E(F F)} \leq 1, f_{E(I F F)} \leq 1\right\}$

Symmetry

Balance

Tool: $\quad$ MATLAB Optimization Toolbox ${ }^{45}$

where $\theta_{k}$ is the fiber angle orientation of each stack of the laminate, $f_{E(F F)}$ and $f_{E(I F F)}$ are respectively fiber and IFF efforts of the laminate. Composite plates are designed in $\left[ \pm \theta_{1} / \pm \theta_{2} / \ldots / \pm \theta_{15} / \pm \theta_{16}\right]_{s}$ sequence by considering ply balance and symmetry constraints. Hence, the number of design variables $\theta_{k}$ reduces from 64 to 16. The proposed hybrid TRRA embedded GA is obtained using predefined operators in MATLAB Optimization Toolbox. ${ }^{45}$

Accordingly, optimum stacking sequence of the composite plate giving maximum buckling strength is searched for the specified design cases in different discrete domains in which one includes integer angle values between $-90^{\circ}$ and $90^{\circ}$, and the other includes $0^{\circ}, \pm 45^{\circ}$ and $90^{\circ}$ discrete values. In addition to the symmetry and balance geometric constraints, PFC (fiber and inter-fiber) are implemented as the first ply failure constraints to the optimization. Thus, the reliability of the designs related to both buckling and static failure strengths is guaranteed.

\section{Results and discussion}

\section{Algorithm performance}

In order to improve the reliability and to obtain the best results from the HA, it is necessary to determine the appropriate options for both sub-algorithms.
Table 2. Specifications of the test problem I.

\begin{tabular}{ll}
\hline Geometrical & $a=508 \mathrm{~mm}, t_{\text {ply }}=0.127 \mathrm{~mm}$, \\
& $\mathrm{NL}=64$ layers \\
Material properties & $E_{1}=127.6 \mathrm{GPa}, E_{2}=13.0 \mathrm{GPa}$, \\
& $G_{12}=6.4 \mathrm{GPa}, v_{12}=0.3$ \\
Loading & $N_{x}=175 \mathrm{~N} / \mathrm{m}$ \\
\hline
\end{tabular}

Table 3. Design cases for the test problem I.

\begin{tabular}{lll}
\hline Design case & $a / b$ & $N_{x} / N_{y}$ \\
\hline DCI & 2 & 2 \\
DC2 & 0.5 & 2 \\
DC3 & 2 & 0.5 \\
DC4 & 0.5 & 0.5 \\
\hline
\end{tabular}

Hence, a preliminary study was performed to understand which parameters would yield the most accurate results in the optimization, and the most appropriate options for GA were determined as follows: population type of double vector, population size of 40 , population creation function of constraint dependent, and initial range of [-90;90]; fitness scaling function of rank; selection function of roulette; reproduction with a crossover fraction of 0.8 with a scattered function, and elite count of 2; a constraint dependent mutation function; generations of 1000 as stopping criteria.

TRRA parameters are taken to be a forward difference to approximate the derivatives, relative perturbation vector $\mathrm{r}$ of sqrt(eps)*ones(numberOf Variables,1), minimum perturbation of 0 , and maximum perturbation of Inf; hessian sparsity pattern of sparse(ones(numberOfVariables)), and no hessian multiply function for user-supplied derivatives; preconditioned conjugate gradient sub-problem algorithm with preconditioner bandwidth of 0 , and typical magnitude of the variables of 1 ; maximum iterations of 8 , and tolerance of 0.1 as inner iteration stopping criteria; maximum iterations of 400 , maximum function evaluations of 1600 , termination tolerance for the variables of $10^{-6}$, and function tolerance of $10^{-6}$ as stopping criteria.

An optimization problem previously studied by the others $^{5,8,13,14}$ was considered as test problem 1 and solved with the selected options to assure the effectiveness and the reliability of the HA in terms of finding global optima. Specifications of the test problem 1 are given in Table 2.

The design cases which include both single and multiple global optima are considered for the optimization as in a previous study, ${ }^{13}$ and these cases selected for the test problem 1 are specified in Table 3 . 
Table 4. Comparison results of the test problem I in terms of finding global optima.

\begin{tabular}{|c|c|c|c|c|c|}
\hline \multirow[b]{2}{*}{ Design case } & \multirow[b]{2}{*}{ One global optimum } & \multirow[b]{2}{*}{$\lambda_{c b}$} & \multicolumn{3}{|c|}{ Number of global optima } \\
\hline & & & $\mathrm{GA}^{\text {present }}$ & $\mathrm{HA}^{\text {present }}$ & $\mathrm{GA}^{13}$ \\
\hline $\mathrm{DCl}$ & {$\left[90_{2} / \pm 45_{3} / 90_{8} / \pm 45_{5} / 90_{6}\right]_{s}$} & $\mathrm{I}, 057,948.3$ & $4 \mid(985 s)$ & 44 (1065 s) & 16 \\
\hline DC2 & {$\left[0_{16} / \pm 45 / 0_{6} /\left( \pm 45 / 0_{2}\right)_{s}\right]_{s}$} & $206,492.9$ & I (102| s) & I (1025 s) & 1 \\
\hline DC3 & {$\left[90_{16} / \pm 45 / 90_{6} /\left( \pm 45 / 90_{2}\right)_{s}\right]_{s}$} & $4 \mid 2,985.8$ & I (1089s) & I (109| s) & 1 \\
\hline DC4 & {$\left[0_{2} / \pm 45_{3} / 0_{8} / \pm 45_{5} / 0_{6}\right]_{5}$} & $132,243.5$ & $36(1020 s)$ & $42(1066 s)$ & 18 \\
\hline
\end{tabular}

Table 5. Details of the load cases for test problem 2.

\begin{tabular}{|c|c|c|c|c|c|}
\hline Load case & $\begin{array}{l}\text { Number } \\
\text { of plies }\end{array}$ & $a(\mathrm{~cm})$ & $b(\mathrm{~cm})$ & $N_{x}(\mathrm{~N} / \mathrm{cm})$ & $N_{y}(\mathrm{~N} / \mathrm{cm})$ \\
\hline I & 48 & 50.8 & 12.7 & 1.75 & 0.22 \\
\hline 2 & 48 & 50.8 & 12.7 & 1.75 & 0.44 \\
\hline 3 & 48 & 50.8 & 12.7 & 1.75 & 0.88 \\
\hline 4 & 64 & 50.8 & 25.4 & 1.75 & 1.75 \\
\hline
\end{tabular}

The optimization is separately performed by using GA and hybrid genetic algorithm (HA). Both algorithms are run 200 times with random starting points as indicated in the reference study. The numbers of distinct global optima for the cases are compared to those of the previous study ${ }^{13}$ as given in Table 4 . Furthermore, average CPU times required by GA and HA separately are indicated in brackets for each design case in the table. Optimization is carried out using a personal computer with a quad core $3.40 \mathrm{GHz}$ processor, and the average CPU time is calculated for ten independent searches.

The results of the test problem 1 indicate that the proposed hybrid GA with the predefined options yields more number of solutions in the design cases, which have multiple solutions when compared to the GA results. It is also seen from Table 4 that the average CPU times do not show large changes in the design cases. However, average computer time calculated for the HA increases in DC1 and DC4 as the HA runs the TRRA sub-algorithm after GA terminates for each iteration. It can be concluded that the proposed HA works more efficiently in finding global optima in the problems which have multiple solutions considering the single performances of the GA and the reference GA.

In addition to the comparison study of global optimum numbers, another problem previously discussed $d^{5,8}$ is considered as test problem 2 to evaluate the performance of the HA in terms of best stacking sequences giving maximum critical buckling load factor by comparing the results with the best known results in the literature. ${ }^{17,20,24,25}$ Table 5 shows the details of composite plate dimensions ( $a$ and $b$ ) and in-plane loads $\left(N_{x}\right.$ and $\left.N_{y}\right)$ for the load cases. In the literature, these load cases have been solved using different HAs. The optimum critical buckling load factors for all the four load cases are compiled from the literature and presented together with our results in Table 6 .

Table 6 shows the optimum stacking sequences obtained using the proposed hybrid GA and their corresponding critical buckling load factors. It should be noted that the buckling load factor results are given in British units to provide consistency with the literature. Three results are shown for each load case. In the first three design cases, ply contiguity constraint is applied for the stacking sequences, which means that maximum number of contiguous plies of the same orientation is constrained to four. Apart from this, stacking sequences are subjected to symmetry and balance constraints. In order to provide an average quality of solutions, each load case is performed 100 times with different starting points. It can be seen from Table 6 that the optimum stacking sequences having superior critical buckling load factors compared to the literature are obtained for load cases 1, 2, and 3 using the proposed hybrid trust region reflective-based GA. It may be noted that the results reported in the literature for load case 4 are without ply contiguity constraint. Therefore, the results for load case 4 are obtained without ply contiguity constraint. Nevertheless, the critical buckling load factor results for the load case 4 are lower than the results reported in the literature. In the present study, it is observed in fact that the optimum stacking sequences found in the reference studies ${ }^{17,20,24,25}$ are obtained with the critical buckling load factor value of 3973.01 as reported in the reference study. $5,8,13$

\section{Effect of failure criteria}

In this section, the effect of using different failure criteria as first ply failure constraints on the buckling optimization of composite laminates is investigated. The aim is to check the accuracy of the results obtained using simple traditional failure criteria $\left(\mathrm{TW}^{46}\right.$ and Hashin-Rotem $(\mathrm{HR})^{47}$ ) with respect to more complex but accurate failure criteria (Puck). 
Table 6. Performance results of the hybrid genetic algorithm.

\begin{tabular}{|c|c|c|c|}
\hline Load case & $\lambda_{c b}^{17,20,24,25}$ & $\lambda_{c b}^{\text {present }}$ & Stacking sequence ${ }^{\text {present }}$ \\
\hline \multirow[t]{3}{*}{ I } & $16,120.38$ & $21,678.75$ & {$\left[90_{4} /\left( \pm 45 / 90_{2}\right)_{2} / \pm 45_{2} / 90_{4} / \pm 45_{2}\right]_{s}$} \\
\hline & $16,119.48$ & $21,437.45$ & {$\left[90_{4} /\left( \pm 45 / 90_{2}\right)_{2} / \pm 45_{6}\right]_{\mathrm{s}}$} \\
\hline & $16,087.83$ & $21,312.49$ & {$\left[90_{4} / \pm 45 / 90_{2} / \pm 45_{3} / 90_{4} / \pm 45_{3}\right]_{s}$} \\
\hline \multirow[t]{3}{*}{2} & $13,442.04$ & $15,984.37$ & {$\left[\left(90_{4} / \pm 45_{2}\right)_{2} / \pm 45 / 90_{2} / \pm 45_{2}\right]_{s}$} \\
\hline & $|3,44| .28$ & $15,690.29$ & {$\left[90_{2} /\left(90_{2} / \pm 45\right)_{2} / \pm 45_{5} / 90_{2} / \pm 45\right]_{s}$} \\
\hline & $13,435.94$ & $15,512.55$ & {$\left[90_{4} / \pm 45_{2} / 90_{2} / \pm 45_{7}\right]_{s}$} \\
\hline \multirow[t]{3}{*}{3} & $10,003.53$ & $10,445.11$ & {$\left[90_{2} /\left(90_{2} / \pm 45\right)_{2} / \pm 45_{7}\right]_{\mathrm{s}}$} \\
\hline & $10,002.95$ & $10,343.85$ & {$\left[90_{4} / \pm 45_{2} / 90_{2} / \pm 45_{6} / 90_{2}\right]_{s}$} \\
\hline & 9999.45 & $10,341.70$ & {$\left[90_{4} / \pm 45_{2} / 90_{2} / \pm 45_{7}\right]_{s}$} \\
\hline \multirow[t]{3}{*}{4} & 3977.12 & 3973.01 & {$\left[ \pm 45 / 90_{10} / \pm 45 / 90_{8} / \pm 45 / 90_{8}\right]_{\mathrm{s}}$} \\
\hline & 3977.12 & 3973.01 & {$\left[90_{2} / \pm 45 / 90_{6} / \pm 45 / 90_{8} / \pm 45 / 90_{10}\right]_{s}$} \\
\hline & 3977.12 & 3973.01 & {$\left[90_{8} /\left( \pm 45 / 90_{2}\right)_{3} / \pm 45_{6}\right]_{s}$} \\
\hline
\end{tabular}

Table 7. Optimum stacking sequences and the corresponding critical buckling load factors using different failure criteria constraints.

\begin{tabular}{llr}
\hline Failure criteria & Stacking sequence & $\lambda_{c b}$ \\
\hline Tsai-Wu & {$\left[90_{12} / \pm 45 / 90_{2} / 0_{2} / \pm 45 / 90_{2} / \pm 45_{2} / 0_{2} / \pm 45_{2}\right]_{s}$} & 1.8921 \\
Hashin-Rotem & {$\left[90_{10} / 0_{2} / \pm 45 / 90_{4} / \pm 45 / 90_{2} / 0_{4} / \pm 45 / 0_{2} / 90_{2}\right]_{s}$} & 1.8025 \\
Puck & {$\left[90_{8} / \pm 45 / 0_{2} / \pm 45_{2} / 90_{2} /\left(90_{2} / \pm 45\right)_{2} / 0_{6}\right]_{s}$} & 1.7380 \\
\hline
\end{tabular}

A composite plate as shown in Figure 3 having an aspect ratio of 4 is loaded such that $N_{x}=10,000 \mathrm{~N} / \mathrm{mm}$ with a load ratio of 1 . The plate is optimized as specified in the Problem Definition section. But here only conventional fiber angles $\left(0^{\circ},+45^{\circ},-45^{\circ}\right.$ and $\left.90^{\circ}\right)$ are used for stacking sequence design. Besides, it is already reported that dispersing the fiber angles can yield damage tolerant stacking sequence designs. ${ }^{48}$ The results obtained using different failure criteria are presented in Table 7. Optimization is performed at least 50 times for each failure criterion to provide consistency.

It can be clearly seen from the table that different optimum stacking sequence designs and critical buckling load factor values are found. Note that the critical buckling load factor values decrease when the criterion used as constraint for the optimization problem becomes more complicated and physically based. For a better understanding of the differences between the predictions of critical buckling load factor values and the stacking sequences for the failure criteria, the optimum solutions for the related design case are compared by considering failure envelopes corresponding to each failure criterion.

Figure 4 shows the optimum solutions with the failure boundaries of the three failure criteria at the critical stress levels. A close look at the figure indicates that the
OD obtained by considering TW and HR failure criteria are out of feasible region based on the Puck failure criterion (Puck line). When different loading cases are examined for the optimization, it is also seen that optimum solutions under the each of the constraints TW and HR failure criteria are not reliable compared to PFC. Based on these results, PFC can be selected as the first ply failure constraint for the remaining analyzes.

\section{Buckling optimization with PFC}

In this section, results of optimum stacking sequence designs with possible highest buckling strength obtained considering Puck fiber and IFF criteria constraints are presented for different design cases. 128 different design problems have been treated in the study considering the previously stated loadings, load ratios and aspect ratios. Details of these design problems with different load and plate aspect ratios are given in Table 8.

In Tables 9 to 12, the obtained optimum stacking sequence designs with integer (Int.) and discrete (Disc.) fiber angle configuration types, the maximized critical buckling load factors $\left(\lambda_{c b}\right)$ and the corresponding fiber and IFF efforts $\left(f_{E(F F)}, f_{E(I F F)}\right)$ are presented for the specified design cases. 


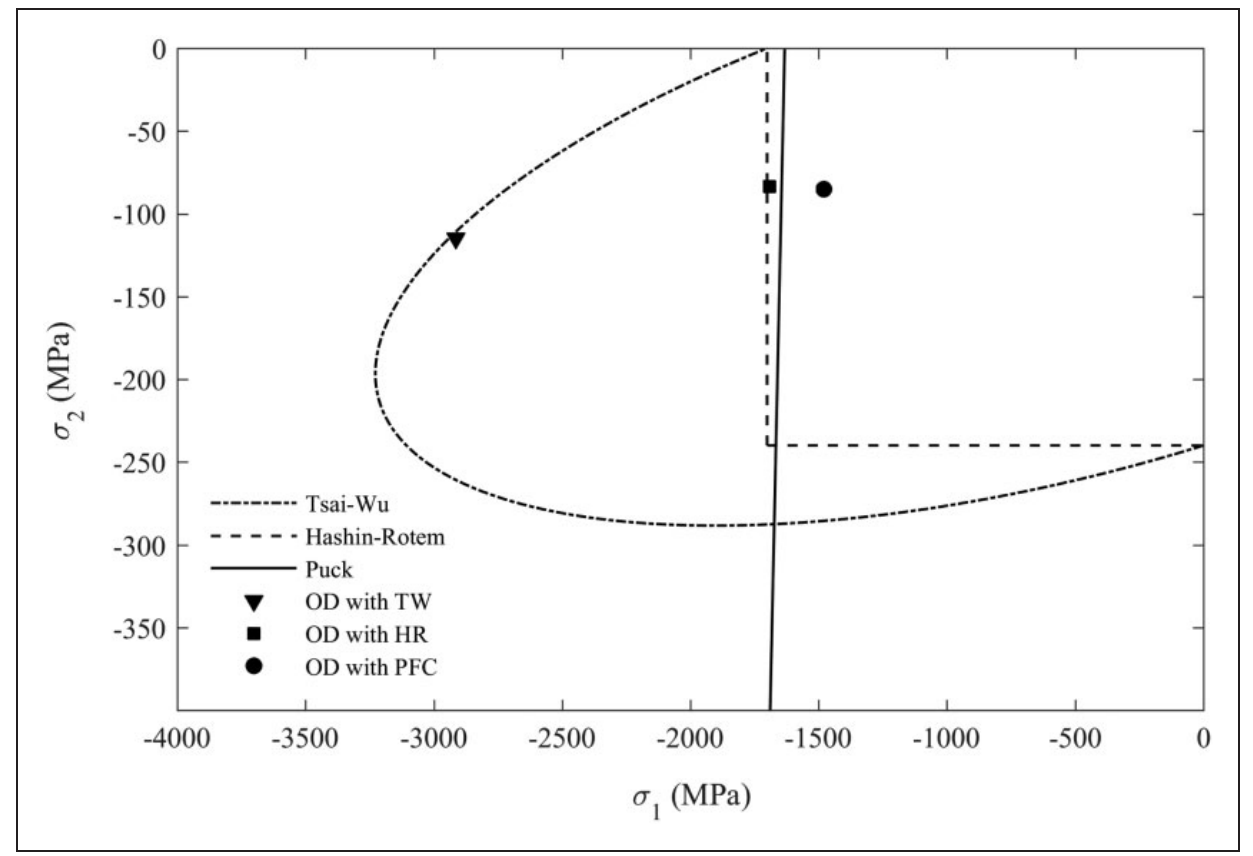

Figure 4. Optimum Designs (OD) with respect to different failure criteria.

Table 8. Composite plate design cases.

\begin{tabular}{llc}
\hline Design case $(D C)$ & Load ratio $\left(N_{x} / N_{y}\right)$ & Aspect ratio $(a / b)$ \\
\hline Ia & $\mathrm{I} / 2$ & $\mathrm{I} / 2$ \\
$\mathrm{Ib}$ & $\mathrm{I} / 2$ & $\mathrm{I}$ \\
$\mathrm{IC}$ & $\mathrm{I} / 2$ & 2 \\
$\mathrm{Id}$ & $\mathrm{I} / 2$ & 4 \\
$\mathrm{2a}$ & $\mathrm{I}$ & $\mathrm{I} / 2$ \\
$\mathrm{2b}$ & $\mathrm{I}$ & $\mathrm{I}$ \\
$2 \mathrm{c}$ & $\mathrm{I}$ & 2 \\
$2 \mathrm{~d}$ & $\mathrm{I}$ & 4 \\
$3 \mathrm{a}$ & 2 & $\mathrm{I} / 2$ \\
$3 \mathrm{~b}$ & 2 & $\mathrm{I}$ \\
$3 \mathrm{c}$ & 2 & 2 \\
$3 \mathrm{~d}$ & 2 & 4 \\
$4 \mathrm{a}$ & 4 & $\mathrm{I} / 2$ \\
$4 \mathrm{~b}$ & 4 & $\mathrm{I}$ \\
$4 \mathrm{c}$ & 4 & 2 \\
$4 \mathrm{~d}$ & 4 & 4 \\
\hline
\end{tabular}

Table 9 shows the results for $N_{x}=1000 \mathrm{~N} / \mathrm{mm}$ loading. In almost all design cases, an optimum reliable stacking sequence design is found. It is observed that the determined critical buckling load factor values of the plates increase when both plate aspect ratio and load ratio increase. Consequently, the plates having the highest buckling strength are obtained for design cases $4 \mathrm{a}-\mathrm{d}$. This trend is valid for all the other loading cases of $N_{x}$. Higher buckling strength is obtained in the integer composite plate configurations compared to the discrete ones. For the design cases $1-4 \mathrm{~b}, 4 \mathrm{a}$, and $1-4 \mathrm{~d}$, similar stacking sequence designs are obtained with the same critical buckling load factor and failure effort values for both integer and discrete type of designs (e.g. $\left[0_{32}\right]_{s},\left[90_{32}\right]_{s}$ ). It is also seen that in design cases $1-4 \mathrm{~b}$, the difference in the stacking of $+45^{\circ}$ and $-45^{\circ}$ angles has not any effect on the results.

In Table 10, the optimum results for $N_{x}=3000$ $\mathrm{N} / \mathrm{mm}$ loading are given. It is seen that the number of ODs decreases with loading increase. For both integer and discrete types of design cases $1-4 \mathrm{~d}$, only $\left[90_{32}\right]_{S}$ stacking sequence is obtained, which satisfies the highest buckling strength. In the cases of $2 \mathrm{c}, 3 \mathrm{c}$ and $4 \mathrm{c}$, multiaxial configurations of the laminates are found, and as might be expected, integer-angled stacking sequences have more buckling load-carrying capacity than the discrete ones.

Table 11 shows the results for $5000 \mathrm{~N} / \mathrm{mm}$ loading. Optimum solutions are obtained for six design cases in both integer and discrete angle types. All the stacking sequences are seen to be in multiaxial configurations. It is understood from the failure effort values that in integer domain the algorithm finds the solutions within failure limits $\left(f_{E(F F)} \leq 1\right.$ or $\left.f_{E(I F F)} \leq 1\right)$ to achieve higher buckling strength.

In Table 12, the optimization results of $N_{x}=10,000$ $\mathrm{N} / \mathrm{mm}$ loading are presented. It is seen from the results that the ODs can be achieved in the boundaries of Puck failure as $f_{E(F F)} \leq 1$ or $f_{E(F F)} \approx 1$ under these high inplane compressive loads. Normally, it is very hard or impossible to find optimum stacking sequence in such 
Table 9. Optimum stacking sequence designs and the corresponding failure efforts for $N_{x}=1000 \mathrm{~N} / \mathrm{mm}$ loading.

\begin{tabular}{|c|c|c|c|c|c|}
\hline DC & Type & Stacking sequence & $\lambda_{c b}$ & $f_{E(F F)}$ & $f_{E(I F F)}$ \\
\hline \multirow[t]{2}{*}{ Ib } & Int. & {$\left[ \pm 45_{2} / \mp 45 / \pm 45_{2} / \mp 45_{2} / \pm 45_{3} / \mp 45_{3} / \pm 45_{3}\right]_{s}$} & I.0867 & 0.1055 & 0.2657 \\
\hline & Disc. & {$\left[ \pm 45_{\mid 6}\right]_{s}$} & I.0867 & 0.1055 & 0.2657 \\
\hline \multirow[t]{2}{*}{ Ic } & Int. & $\begin{array}{l}{\left[90_{2} / \pm 80 / \mp 78 / \mp 77 / \mp 81 / \mp 79 / \mp 73 / \mp 79 /\right.} \\
\left. \pm 79 / \pm 68 / \mp 83 / \mp 70 / 90_{2} / \mp 75 / \mp 60 / \pm 31\right]_{s}\end{array}$ & 2.7930 & 0.2567 & 0.1536 \\
\hline & Disc. & {$\left[90_{12} / \pm 45 / 90_{6} / \pm 45_{3} / 90_{2} / \pm 45_{2}\right]_{s}$} & 2.7433 & 0.1383 & 0.0986 \\
\hline \multirow[t]{2}{*}{ Id } & Int. & {$\left[90_{32}\right]_{s}$} & 11.4049 & $0.07 \mid I$ & 0.2604 \\
\hline & Disc. & {$\left[90_{32}\right]_{s}$} & 11.4049 & $0.07 \mid I$ & 0.2604 \\
\hline \multirow[t]{2}{*}{$2 \mathrm{a}$} & Int. & $\begin{array}{l}{[ \pm 21 / \pm 23 / \pm 19 / \mp 21 / \pm 9 / \pm 27 / \pm 11 / \pm 18 /} \\
\mp 20 / \mp 14 / \mp 11 / \pm 4 / \mp 14 / \pm 34 / \pm 7 / \mp 42]_{s}\end{array}$ & 1.2096 & 0.1935 & 0.1962 \\
\hline & Disc. & {$\left[ \pm 45 / 0_{8} / \pm 45 / 0_{6} /\left( \pm 45 / 0_{2}\right)_{2} / 0_{6}\right]_{s}$} & I.1567 & 0.1604 & 0.1368 \\
\hline \multirow[t]{2}{*}{$2 b$} & Int. & {$\left[\mp 45 /\left( \pm 45 / \mp 45_{2}\right)_{2} / \pm 45 / \mp 45 / \pm 45_{2} / \mp 45 / \pm 45_{2} / \mp 45_{2}\right]_{s}$} & 1.6301 & 0.0699 & 0.0395 \\
\hline & Disc. & {$\left[ \pm 45_{16}\right]_{s}$} & 1.6301 & 0.0699 & 0.0395 \\
\hline \multirow[t]{2}{*}{$2 c$} & Int. & $\begin{array}{l}{[\mp 72 / \pm 74 / \mp 70 / \mp 68 / \mp 71 / \mp 73 / \mp 70 / \pm 71 /} \\
\mp 70 / \mp 69 / \mp 69 / \mp 70 / \pm 67 / \pm 50 / \pm 75 / \mp 86]_{s}\end{array}$ & 4.8382 & 0.1904 & 0.2152 \\
\hline & Disc. & {$\left[90_{8} / \pm 45_{2} / 90_{4} / \pm 45_{8}\right]_{s}$} & 4.6268 & 0.0943 & 0.0782 \\
\hline \multirow[t]{2}{*}{$2 d$} & Int. & {$\left[90_{32}\right]_{s}$} & 20.5288 & 0.0329 & 0.2604 \\
\hline & Disc. & {$\left[90_{32}\right]_{s}$} & 20.5288 & 0.0329 & 0.2604 \\
\hline \multirow[t]{2}{*}{$3 a$} & Int. & $\begin{array}{l}{\left[ \pm 13 / \pm 9 / \pm 8 / \mp 11 / \pm 9 / \mp 15 / \pm 1 I_{2} / \mp 17 /\right.} \\
\left.\mp 13 / \pm 10 / \mp 16 / \pm 10_{2} / \pm 11 / \pm 8\right]_{s}\end{array}$ & 1.3989 & $0.048 I$ & 0.1210 \\
\hline & Disc. & {$\left[0_{6} / \pm 45 / 0_{22} / \pm 45\right]_{s}$} & 1.3721 & 0.1102 & 0.0875 \\
\hline \multirow[t]{2}{*}{$3 b$} & Int. & {$\left[\mp 45 / \pm 45 / \mp 45_{2} / \pm 45 / \mp 45 / \pm 45_{2} / \mp 45 / \pm 45 / \mp 45 / \pm 45_{2} / \mp 45_{2} / \pm 45_{2] s}\right.$} & 2.1735 & 0.0526 & 0.1328 \\
\hline & Disc. & {$\left[ \pm 45_{16}\right]_{s}$} & 2.1735 & 0.0526 & 0.1328 \\
\hline \multirow[t]{2}{*}{$3 c$} & Int. & $\begin{array}{l}{\left[( \pm 63 / \mp 62)_{2} / \mp 62 / \pm 63 / \pm 61 / \pm 63 / \mp 63 /\right.} \\
\left.\mp 62 / \mp 61 / \pm 60 / \pm 57 / \mp 70 / \pm 56 / 90_{2}\right]_{s}\end{array}$ & 7.5184 & 0.1089 & 0.2278 \\
\hline & Disc. & {$\left[\left( \pm 45_{2} / 90_{4}\right)_{2} / 90_{2} / \pm 45 / 90_{4} /\left(90_{2} / \pm 45\right)_{2}\right]_{s}$} & 7.0417 & 0.1145 & 0.1070 \\
\hline \multirow[t]{2}{*}{$3 d$} & Int. & {$\left[90_{32}\right]_{s}$} & 34.2147 & 0.0137 & 0.2604 \\
\hline & Disc. & {$\left[90_{32}\right]_{s}$} & 34.2147 & 0.0137 & 0.2604 \\
\hline \multirow[t]{2}{*}{$4 a$} & Int. & {$\left[0_{32}\right]_{s}$} & 1.5095 & 0.0369 & 0.0651 \\
\hline & Disc. & {$\left[0_{32}\right]_{s}$} & 1.5095 & 0.0369 & 0.0651 \\
\hline \multirow[t]{2}{*}{$4 b$} & Int. & {$\left[ \pm 45 / \mp 45 / \pm 45_{2} / \mp 45 / \pm 45_{3} / \mp 45_{4} / \pm 45 / \mp 45_{3}\right]_{s}$} & 2.6082 & 0.0440 & 0.2095 \\
\hline & Disc. & {$\left[ \pm 45_{16}\right]_{s}$} & 2.6082 & 0.0440 & 0.2095 \\
\hline \multirow[t]{2}{*}{$4 c$} & Int. & $\begin{array}{l}{\left[\mp 53_{2} /( \pm 53 / \mp 53)_{2} / \mp 53 / \pm 53 / \mp 53_{2} /\right.} \\
\left. \pm 53 / \mp 53_{2} / \pm 59 / \mp 51 / \pm 80\right]_{s}\end{array}$ & 10.0513 & 0.1930 & 0.1971 \\
\hline & Disc. & {$\left[ \pm 45_{4} / 90_{4} / \pm 45_{5} / 90_{8} \pm 45\right]_{s}$} & 9.5280 & 0.0878 & 0.0945 \\
\hline \multirow[t]{2}{*}{$4 d$} & Int. & {$\left[90_{32}\right]_{s}$} & 51.3220 & 0.0041 & 0.2604 \\
\hline & Disc. & {$\left[90_{32}\right]_{s}$} & 51.3220 & $0.004 I$ & 0.2604 \\
\hline
\end{tabular}


Table 10. Optimum stacking sequence designs and the corresponding failure efforts for $N_{x}=3000 \mathrm{~N} / \mathrm{mm}$ loading.

\begin{tabular}{|c|c|c|c|c|c|}
\hline DC & Type & Stacking sequence & $\lambda_{c b}$ & $f_{E(F F)}$ & $f_{E(I F F)}$ \\
\hline \multirow[t]{2}{*}{ Id } & Int. & {$\left[90_{32}\right]_{s}$} & 3.8016 & 0.2134 & 0.7813 \\
\hline & Disc. & {$\left[90_{32}\right]_{s}$} & 3.8016 & 0.2134 & 0.7813 \\
\hline \multirow[t]{2}{*}{$2 c$} & Int. & $\begin{array}{l}{[\mp 68 / \pm 72 / \pm 69 / \mp 70 / \pm 69 / \pm 72 / \mp 78 / \pm 66 /} \\
\left. \pm 77 / \mp 74 / 90_{2} / \mp 79 / \mp 58 / \mp 71 / 90_{2} / \pm 28\right]_{s}\end{array}$ & 1.6146 & 0.7966 & 0.4571 \\
\hline & Disc. & {$\left[90_{8} / \pm 45_{2} / 90_{4} / \pm 45_{8}\right]_{s}$} & 1.5423 & 0.2831 & 0.2347 \\
\hline \multirow[t]{2}{*}{$2 d$} & Int. & {$\left[90_{32}\right]_{s}$} & 6.8429 & 0.0986 & 0.7813 \\
\hline & Disc. & {$\left[90_{32}\right]_{s}$} & 6.8429 & 0.0986 & 0.7813 \\
\hline \multirow[t]{2}{*}{$3 c$} & Int. & $\begin{array}{l}{[ \pm 62 / \mp 64 / \mp 63 / \pm 61 / \pm 64 / \mp 62 / \mp 63 / \pm 61 /} \\
\mp 61 / \pm 62 / \mp 61 / \pm 58 / \mp 68 / \pm 57 / \pm 49 / \pm 32]_{s}\end{array}$ & 2.5028 & 0.7587 & 0.4512 \\
\hline & Disc. & {$\left[ \pm 45_{3} / 90_{4} /\left(90_{4} / \pm 45\right)_{3} / 90_{2} / 45\right]_{s}$} & 2.3472 & 0.3438 & 0.3209 \\
\hline \multirow[t]{2}{*}{$3 d$} & Int. & {$\left[90_{32}\right]_{s}$} & II.4049 & 0.0412 & 0.7813 \\
\hline & Disc. & {$\left[90_{32}\right]_{s}$} & II.4049 & 0.0412 & 0.7813 \\
\hline \multirow[t]{2}{*}{$4 c$} & Int. & $\begin{array}{l}{\left[ \pm 54 /(\mp 53 / \pm 53)_{2} / \mp 54 / \pm 54 / \pm 51 / \pm 52 /\right.} \\
\mp 52 / \mp 54 / \pm 55 / \mp 50 / \mp 52 / \pm 65 / \pm 71]_{s}\end{array}$ & 3.3583 & 0.4331 & 0.5876 \\
\hline & Disc. & {$\left[ \pm 45_{4} / 90_{2} / \pm 45 /\left(90_{2} / \pm 45_{2}\right)_{2} / 90_{4} / \pm 45 / 90_{2}\right]_{s}$} & 3.1760 & 0.2635 & 0.2836 \\
\hline \multirow[t]{2}{*}{$4 d$} & Int. & {$\left[90_{32}\right]_{s}$} & 17.1073 & 0.0124 & 0.7813 \\
\hline & Disc. & {$\left[90_{32}\right]_{s}$} & 17.1073 & 0.0124 & 0.7813 \\
\hline
\end{tabular}

Table II. Optimum stacking sequence designs and the corresponding failure efforts for $N_{x}=5000 \mathrm{~N} / \mathrm{mm}$ loading.

\begin{tabular}{|c|c|c|c|c|c|}
\hline DC & Type & Stacking sequence & $\lambda_{c b}$ & $f_{E(F F)}$ & $f_{E(I F F)}$ \\
\hline \multirow[t]{2}{*}{ Id } & Int. & {$\left[90_{18} / \mp 85 / 90_{2} / \pm 88 / \pm 76 / \mp 49 / \pm 49 / \mp 49\right]_{s}$} & $2.276 \mathrm{I}$ & $0.944 I$ & 0.9937 \\
\hline & Disc. & {$\left[90_{6} / 0_{2} / 90_{12} / 0_{4} / 90_{8}\right]_{s}$} & 1.9921 & 0.7633 & 0.3992 \\
\hline \multirow[t]{2}{*}{$2 d$} & Int. & {$\left[90_{22} / \pm 82 / \pm 68 / \mp 54 / \pm 14 / \mp 6\right]_{s}$} & 4.0924 & 0.9985 & 0.6543 \\
\hline & Disc. & {$\left[90_{24} / \pm 45_{4}\right]_{s}$} & 4.0849 & 0.8028 & 0.6839 \\
\hline \multirow[t]{2}{*}{$3 c$} & Int. & $\begin{array}{l}{\left[ \pm 61 / \pm 65_{2} / \pm 62 / \mp 64 / \mp 62 / \pm 59 / \mp 61 /\right.} \\
\left.\mp 60_{2} / \pm 61 / \pm 51 / \pm 59 / \pm 52 / \pm 44 / \pm 69\right]_{s}\end{array}$ & 1.5050 & 0.9288 & I.004 I \\
\hline & Disc. & {$\left[90_{2} / \pm 45_{2} / 90_{4} / \pm 45_{4} / 90_{4} / \pm 45 / 90_{8}\right]_{s}$} & 1.4083 & 0.5734 & 0.5348 \\
\hline \multirow[t]{2}{*}{$3 d$} & Int. & {$\left[90_{26} / \mp 58 / \mp 49_{2}\right]_{s}$} & 6.8343 & 0.9060 & 0.9962 \\
\hline & Disc. & {$\left[90_{24} / \pm 45_{2} / 90_{2} / \pm 45\right]_{s}$} & 6.8120 & 0.8857 & 0.8069 \\
\hline \multirow[t]{2}{*}{$4 c$} & Int. & $\begin{array}{l}{\left[\mp 53 / \mp 54 / \mp 53 / \pm 54_{3} / \pm 51 / \mp 53_{2} /\right.} \\
\pm 55 / \pm 54 / \mp 49 / \pm 50 / \pm 47 / \mp 42 / \pm 67]_{s}\end{array}$ & 2.0147 & 0.8251 & 0.9997 \\
\hline & Disc. & {$\left[ \pm 45_{2} / 90_{2} / \pm 45_{6} / 90_{2} /\left(90_{4} / \pm 45\right)_{2}\right]_{s}$} & 1.9056 & 0.4395 & 0.4726 \\
\hline \multirow[t]{2}{*}{$4 d$} & Int. & {$\left[90_{26} / \pm 60 / \mp 5 \mathrm{I} / \pm 50\right]_{s}$} & 10.2529 & 0.8860 & 1.0083 \\
\hline & Disc. & {$\left[90_{20} / \pm 45 / 90_{4} / \pm 45_{2} / 90_{2}\right]_{s}$} & 10.1691 & 0.8737 & 0.8007 \\
\hline
\end{tabular}


Table 12. Optimum stacking sequence designs and the corresponding failure efforts for $N_{x}=10,000 \mathrm{~N} / \mathrm{mm}$ loading.

\begin{tabular}{|c|c|c|c|c|c|}
\hline DC & Type & Stacking sequence & $\lambda_{c b}$ & $f_{E(F F)}$ & $f_{E(I F F)}$ \\
\hline \multirow[t]{2}{*}{$2 d$} & Int. & {$\left[90_{16} / \mp 82 / 90_{2} / \pm 15 / \pm 45 / \pm 22 / \mp 2 / \pm 2\right]_{s}$} & 1.9810 & 0.9996 & $0.8 \mid 40$ \\
\hline & Disc. & {$\left[90_{8} / \pm 45 / 0_{2} / \pm 45_{2} / 90_{2} /\left(90_{2} / \pm 45\right)_{2} / 0_{6}\right]_{s}$} & 1.7380 & 0.8985 & 0.5980 \\
\hline \multirow[t]{2}{*}{$3 d$} & Int. & {$\left[90_{18} / \pm 84 / \pm 4 \mathrm{I} / \pm 4 / \mp 4 / \pm 5 / \mp 4 / \pm 4\right]_{s}$} & 3.3051 & 0.9999 & 0.6829 \\
\hline & Disc. & {$\left[90_{12} / \pm 45 / 90_{2} / 0_{2} / 90_{4} /\left(0_{2} / \pm 45\right)_{2} / 0_{2}\right]_{s}$} & 3.1615 & 1.0828 & 0.9468 \\
\hline $4 c$ & Int. & $\begin{array}{l}{[\mp 55 / \pm 57 / \pm 56 / \mp 54 / \mp 53 / \mp 51 / \pm 50 /} \\
\pm 49 / \pm 52 / \pm 55 / \pm 43 / \pm 13 / \mp 20 / \mp 6 / \mp 42]_{s}\end{array}$ & 0.9914 & 1.0049 & 0.5056 \\
\hline \multirow[t]{2}{*}{$4 d$} & Int. & {$\left[90_{20} / \mp 39 / 0_{10}\right]_{s}$} & 4.9540 & 1.0012 & 0.6177 \\
\hline & Disc. & {$\left[90_{20} / \pm 45 / 0_{10}\right]_{s}$} & 4.9643 & 1.0193 & 0.6701 \\
\hline
\end{tabular}

Table 13. Comparison of constrained and unconstrained optimization.

\begin{tabular}{|c|c|c|c|c|c|}
\hline DC & Optimization & Stacking sequence & $\lambda_{c b}$ & $f_{E(F F)}$ & $f_{E(I F F)}$ \\
\hline \multirow[t]{2}{*}{$2 d$} & Const. & {$\left[90_{16} / \mp 82 / 90_{2} / \pm 15 / \pm 45 / \pm 22 / \mp 2 / \pm 2\right]_{s}$} & 1.9810 & 0.9996 & 0.8140 \\
\hline & Unconst. & {$\left[90_{32}\right]_{s}$} & 2.0529 & 0.3286 & 4.1681 \\
\hline \multirow[t]{2}{*}{$3 d$} & Const. & {$\left[90_{18} / \pm 84 / \pm 4 \mathrm{I} / \pm 4 / \mp 4 / \pm 5 / \mp 4 / \pm 4\right]_{s}$} & 3.3051 & 0.9999 & 0.6829 \\
\hline & Unconst. & {$\left[90_{32}\right]_{s}$} & 3.4215 & 0.1372 & 4.1667 \\
\hline \multirow[t]{2}{*}{$4 c$} & Const. & $\begin{array}{l}{[\mp 55 / \pm 57 / \pm 56 / \mp 54 / \mp 53 / \mp 51 / \pm 50 / \pm 49} \\
\left./ \pm 52 / \pm 55 / \pm 43 / \pm 13 / \mp 20 / \mp 6 / \mp 4_{2}\right]_{s}\end{array}$ & 0.9914 & 1.0049 & 0.5056 \\
\hline & Unconst. & $\begin{array}{l}{\left[ \pm 53 / \pm 55 / \pm 54 / \pm 53 / \pm 56 / \pm 53 / \pm 54 / \pm 5 I_{2}\right.} \\
/ \pm 52 / \pm 51 / \pm 49 / \pm 48 / \pm 45 / \pm 39 / \pm 34]_{s}\end{array}$ & 1.0073 & 2.0820 & 36.0359 \\
\hline \multirow[t]{2}{*}{$4 d$} & Const. & {$\left[90_{20} / \mp 39 / 0_{10}\right]_{s}$} & 4.9540 & 1.0012 & 0.6177 \\
\hline & Unconst. & {$\left[90_{32}\right]_{s}$} & 5.1322 & 0.0414 & 4.1667 \\
\hline
\end{tabular}

excessive loadings. However, the proposed optimization strategy with PFC constraints enables to find feasible solutions. By comparing with the unconstrained optimization, the effectiveness of this strategy is shown in Table 13. In this comparison, only integer type of optimization is considered for $N_{x}=10,000$ $\mathrm{N} / \mathrm{mm}$ loading. As seen from the results, although the critical buckling load bearing capacities of the laminates obtained by unconstrained optimization are higher than those of the constrained optimization, the optimum stacking sequences are not reliable according to the failure effort values of PFC. This situation indicates the importance of implementing complicated failure criteria to buckling optimization problems.

\section{Conclusion}

In the current study, the optimum integer and discrete stacking sequences of symmetric and balanced laminated composites subjected to biaxial compressive loading for maximum buckling load capacity are searched for several design cases. Puck fiber and IFF criteria are implemented to the optimization problems as non-linear constraints. A HA combining GA and TRRA is proposed. First, the two selected test problems with different design cases are solved to examine the performance of the proposed HA, and the results are compared with the published data in the literature. Secondly, the effect of using TW, HR and PFC as constraints on the buckling optimization of composite laminates is investigated by a selected design case. Afterwards, design problems are solved for four different load cases.

Based on the studies carried out, we have concluded with the following results:

1. Regarding the results in the literature, the hybrid TRRA implemented with GA shows superior or at 
least comparable performance to obtain (i) the maximum number of global optima and (ii) critical buckling load factor results in a moderate computational time.

2. In the cases that traditional failure criteria $\mathrm{TW}$ and HR are used as constraints, it is found that the optimum stacking sequence designs which exhibit relatively better buckling strength are not reliable in terms of first ply failure considering PFC constraints.

3. The optimum stacking sequence designs are achieved even in excessive compressive loads $(10,000 \mathrm{~N} / \mathrm{mm})$ by implementing Puck fiber and IFF criteria as nonlinear constraints to the optimization.

4. The comparison study of constrained and unconstrained optimization indicates that the constrained optimization with PFC provides feasible stacking sequence designs that satisfies both buckling and the first ply failure strength requirements, while any feasible design cannot be obtained due to the ply failure in the unconstrained optimization.

In conclusion, the current study underlines the significance of including HAs and physically based failure criteria constraints in buckling optimization problems of laminated composite plates subjected to compressive in-plane loading and gives a better understanding on reliable optimization without first ply failure by the proposed approach.

\section{Declaration of Conflicting Interests}

The author(s) declared no potential conflicts of interest with respect to the research, authorship, and/or publication of this article.

\section{Funding}

The author(s) received no financial support for the research, authorship, and/or publication of this article.

\section{References}

1. Pelletier JL and Vel SS. Multi-objective optimization of fiber reinforced composite laminates for strength, stiffness and minimal mass. Comput Struct 2006; 84: 2065-2080.

2. Ganguli R. Optimal design of composite structures: a historical review. J Indian Inst Sci 2013; 93: 557-570.

3. Ghiasi H, Pasini D and Lessard L. Optimum stacking sequence design of composite materials Part I: constant stiffness design. Compos Struct 2009; 90: 1-11.

4. Ghiasi H, Fayazbakhsh K, Pasini D, et al. Optimum stacking sequence design of composite materials Part II: Variable stiffness design. Compos Struct 2010; 93: 1-13.

5. Le Riche R and Haftka RT. Optimization of laminate stacking sequence for buckling load maximization by genetic algorithm. AIAA J 1993; 31: 951-956.
6. Le Riche R and Haftka RT. Improved genetic algorithm for minimum thickness composite laminate design. Compos Eng 1995; 5: 143-161.

7. Liu B, Haftka RT, Akgün MA, et al. Permutation genetic algorithm for stacking sequence design of composite laminates. Comput Meth Appl Mech Eng 2000; 186: 357-372.

8. Soremekun G, Gürdal Z, Haftka RT, et al. Composite laminate design optimization by genetic algorithm with generalized elitist selection. Comput Struct 2001; 79: 131-143.

9. Park JH, Hwang JH, Lee CS, et al. Stacking sequence design of composite laminates for maximum strength using GAs. Compos Struct 2001; 52: 217-231.

10. Kim CW and Lee JS. Optimal design of laminated composite plates for maximum buckling load using genetic algorithm. Proc Institut Mech EngC J Mech Eng Sci 2005; 219: 869-878.

11. Naik NG, Gopalakrishnan S and Ganguli R. Design optimization of composites using genetic algorithms and failure mechanism based failure criterion. Compos Struct 2008; 83: 354-367.

12. Lopez RH, Luersen MA and Cursi ES. Optimization of laminated composites considering different failure criteria. Compos Part B Eng 2009; 40: 731-740.

13. Karakaya Ş and Soykasap Ö. Buckling optimization of laminated composite plates using genetic algorithm and generalized pattern search algorithm. Struct Multidiscip Optim 2009; 39: 477-486.

14. Erdal O and Sonmez FO. Optimum design of composite laminates for maximum buckling load capacity using simulated annealing. Compos Struct 2005; 71: 45-52.

15. Lakshmi K and Rao ARM. Optimal design of laminate composite plates using dynamic hybrid adaptive harmony search algorithm. J Reinf Plast Compos 2015; 34: 493-518.

16. Pai N, Kaw A and Weng M. Optimization of laminate stacking sequence for failure load maximization using Tabu search. Compos Part B Eng 2003; 34: 405-413.

17. Rao ARM and Arvind N. A scatter search algorithm for stacking sequence optimisation of laminate composites. Compos Struct 2005; 70: 383-402.

18. Aymerich F and Serra M. Optimization of laminate stacking sequence for maximum buckling load using the ant colony optimization (ACO) metaheuristic. Compos Part A Appl S 2008; 39: 262-272.

19. Sebaey TA, Lopes CS, Blanco N, et al. Ant colony optimization for dispersed laminated composite panels under biaxial loading. Compos Struct 2011; 94: 31-36.

20. Rao ARM and Arvind N. Optimal stacking sequence design of laminate composite structures using Tabu embedded simulated annealing. Struct Eng Mech 2007; 25: 239-268.

21. Rao ARM and Shyju PP. Development of a hybrid metaheuristic algorithm for combinatorial optimisation and its application for optimal design of laminated composite cylindrical skirt. Comput Struct 2008; 86: 796-815.

22. Rao ARM and Shyju PP. A Meta-heuristic algorithm for multi-objective optimal design of hybrid laminate 
composite structures. Comput-Aided Civ Inf 2010; 25: 149-170.

23. Rao ARM and Lakshmi K. Discrete hybrid PSO algorithm for design of laminate composites with multiple objectives. J Reinf Plast Compos 2011; 30: 1703-1727.

24. Rao ARM and Lakshmi K. Optimal design of stiffened laminate composite cylinder using a hybrid SFL algorithm. J Compos Mater 2012; 46: 3031-3055.

25. Rao ARM. Lay-up sequence design of laminate composite plates and a cylindrical skirt using ant colony optimization. Proc IMechE, Part G: J Aerospace Eng 2009; 223: 1-18.

26. Puck A and Schürmann H. Failure analysis of FRP laminates by means of physically based phenomenological models. Compos Sci Technol 1998; 58: 1045-1067.

27. Naik GN, Gyan S, Satheesh R, et al. Minimum weight design of fiber-reinforced laminated composite structures with maximum stress and Tsai-Wu failure criteria. $J$ Reinf Plast Compos 2011; 30: 179-192.

28. Soden PD, Hinton MJ and Kaddour AS. A comparison of the predictive capabilities of current failure theories for composite laminates. Compos Sci Technol 1998; 58: 1225-1254.

29. Hinton MJ, Kaddour AS and Soden PD. A comparison of the predictive capabilities of current failure theories for composite laminates, judged against experimental evidence. Compos Sci Technol 2002; 62: 1725-1797.

30. Knops M. Analysis of failure in fiber polymer laminates. Germany: Springer-Verlag, 2008.

31. Aydin L and Artem HS. Comparison of stochastic search optimization algorithms for the laminated composites under mechanical and hygrothermal loadings. J Reinf Plast Compos 2011; 30: 1197-1212.

32. Hwang SF, Wu JC and He RS. Identification of effective elastic constants of composite plates based on a hybrid genetic algorithm. Compos Struct 2009; 90: 217-224.

33. Keller D. Optimization of ply angles in laminated composite structures by a hybrid, asynchronous, parallel evolutionary algorithm. Compos Struct 2010; 92: 2781-2790.

34. Ayesta I, Izquierdo B, Sanchez JA, et al. Optimum electrode path generation for EDM manufacturing of aerospace components. Robot Cim-Int Manuf 2016; 37: 273-281.
35. Ebrahimi B, Hatami T and Vera JH. Use of a hybrid optimization method to reduce vapor-liquid equilibrium data of maverick systems: The case of carbon dioxide with 2-methoxyethanol and 2-ethoxyethanol using cubic equations of state. Fluid Phase Equilibr 2013; 338: 46-53.

36. Syed KS, Iqbal Z and Ishaq M. Optimal configuration of finned annulus in a double pipe with fully developed laminar flow. Appl Therm Eng 2011; 31: 1435-1446.

37. Le TM, Fatahi B and Khabbaz H. Numerical optimisation to obtain elastic viscoplastic model parameters for soft clay. Int J Plast 2015; 65: 1-21.

38. Geletu A. Solving optimization problems using the Matlab Optimization Toolbox - a tutorial, TUIlmenau, Fakultät Math. Ilmenau, Germany: Naturwiss, December 2007.

39. Steihaug T. The conjugate gradient method and trust regions in large scale optimization. SIAM J Numer Anal 1983; 20: 626-637.

40. Branch MA, Coleman TF and Li Y. A subspace, interior, and conjugate gradient method for large-scale boundconstrained minimization problems. SIAM J Sci Comput 1999; 21: 1-23.

41. Vinson JR. Plate and panel structures of isotropic, composite and piezoelectric materials, including sandwich construction. The Netherlands: Springer, 2005.

42. Spallino R and Thierauf G. Thermal buckling optimization of composite laminates by evolution strategies. Comput Struct 2000; 78: 691-697.

43. Gürdal Z, Haftka RT and Hajela P. Design and optimization of laminated composite materials. New York: Wiley, 1999.

44. Gibson RF. Principles of composite material mechanics, 1st ed. USA: Mc-Graw-Hill Inc., 1994.

45. The Mathworks, Inc. MATLAB computer software in version R2008b.

46. Tsai SW and Wu EM. A general theory of strength for anisotropic materials. J Compos Mater 1971; 5: 58-80.

47. Hashin Z and Rotem A. A fatigue failure criterion for fibre reinforced materials. J Compos Mater 1973; 7: 448-464.

48. Gyan S, Ganguli R and Naik GN. Damage-tolerant design optimization of laminated composite structures using dispersion of ply angles by genetic algorithm. J Reinf Plast Compos 2012; 31: 799-814. 